\title{
Optimal design of a plate of variable thickness: a variational approach in dimension one
}

\author{
PABLO PEDREGAL and ALBERTO DONOSO \\ ETSI Industriales, Universidad de Castilla-La Mancha \\ 13071 Ciudad Real, Spain \\ E-mail: adonoso@ind-cr.uclm.es
}

\begin{abstract}
For a typical design problem of a plate of variable thickness, we analyze the onedimensional situation through a variational reformulation to discover that, in contrast with the higher dimensional case, there are optimal solutions. Another typical interpretation of this simplification is that of the optimal shape of a bending beam. The mechanism employed for the existence issue is the direct method for the new formulation. Optimality conditions are then pursued.
\end{abstract}

Mathematical subject classification: 49J15, 49J45, 49K15, 74K20, 74P05.

Key words: periodic plate, optimal design, direct method, optimality conditions.

\section{Introduction}

In this paper, we analyze a simplified, one-dimensional model for the optimal design of a plate of variable thickness which is assumed to be infinite along one of its axes. In general, the Kirchhoff model for pure bending of symmetric plates ([5], [9]) postulates that the deflection of vertical displacement $w$ of a plate is the solution of the fourth order, elliptic equation

$$
\sum_{i, j, k, l} \frac{\partial^{2}}{\partial x_{i} \partial x_{j}}\left(M_{i j k l} \frac{\partial^{2} w}{\partial x_{k} \partial x_{1}}\right)=F \quad \text { in } \Omega,
$$

where $F \in L^{2}(\Omega)$ is the vertical load, $\Omega$ is the midplane of the plate with respect to which the plate is symmetric, and the design of the plate lies in the tensor $M$

$$
M=\frac{2}{3} h^{3}(x) B
$$


where $h$ is the half-thickness and $B$ is the tensor of material constants. Boundary conditions for a clamped plate incorporate

$$
w=\frac{\partial w}{\partial n}=0 \quad \text { on } \partial \Omega .
$$

Under the assumptions that $\Omega=(0,1) \times(0,1)$, both $h$ and $F$ depend only on $x_{1}$, and if we replace the boundary condition $w=0$ on $x_{2}=0, x_{2}=1$, by a periodic boundary condition, it is elementary to check that

$$
w\left(x_{1}, x_{2}\right)=y\left(x_{1}\right)
$$

is the solution of the above problem where $y(x), x=x_{1}$, solves

$$
\begin{gathered}
\left(\frac{2}{3} h^{3}(x) B_{1111} y^{\prime \prime}(x)\right)^{\prime \prime}=F \quad \text { in }(0,1), \\
y(0)=y(1)=y^{\prime}(0)=y^{\prime}(1)=0 .
\end{gathered}
$$

Under these circumstances, the objective is to design the plate, i.e. to choose the function $h(x)$, so that we maximize the overall rigidity of the plate. A way of measuring this global rigidity is through the compliance functional

$$
J(h)=\int_{0}^{1} F(x) y(x) d x
$$

which represents the work done by the load $F$. Hence, maximum global rigidity corresponds to minimum compliance.

Further natural constraints on the feasible designs limit the amount of material to be used

$$
0<h_{-} \leq h(x) \leq h_{+}, \quad \int_{0}^{1} h(x) d x \leq V,
$$

where $h_{-}, h_{+}$and $V$ are positive constants given in a coherent way

$$
h_{-}<V<h_{+} \text {. }
$$

Altogether, and given positive constants $h_{-}<V<h_{+}, B=B_{1111}$ and $F \in L^{2}(0,1)$, we seek to

$$
\text { Minimize } \quad J(h)=\int_{0}^{1} F(x) y(x) d x
$$


where

$$
\begin{aligned}
& \left(\frac{2}{3} h^{3}(x) B y^{\prime \prime}(x)\right)^{\prime \prime}=F \quad \text { in }(0,1), \\
& y(0)=y(1)=y^{\prime}(0)=y^{\prime}(1)=0,
\end{aligned}
$$

and $h$ is feasible as specified in the last paragraph. Notice that the constants $2 / 3$ and $B$ can be incorporated either in the design $h$ or in the load $F$ so that we will look at the somewhat simpler problem

$$
\text { Minimize } \quad J(h)=\int_{0}^{1} F(x) y(x) d x
$$

subject to

$$
\begin{gathered}
\left(h^{3}(x) y^{\prime \prime}(x)\right)^{\prime \prime}=F \quad \text { in }(0,1), \\
y(0)=y(1)=y^{\prime}(0)=y^{\prime}(1)=0,
\end{gathered}
$$

and $h$ is such that

$$
0<h_{-} \leq h(x) \leq h_{+}, \quad \int_{0}^{1} h(x) d x \leq V .
$$

This type of optimal design problems have been analyzed in a number of papers (in addition to the references already cited see [4]) even in the two dimensional situation but the design still depending on one variable. One of the main features of these problems is the lack of optimal designs and the infinitesimal spatial oscillations leading to optimality. In the present work, and as a previous step to the higher dimensional situation, we pretend to recast the above optimal control problem in a purely variational format that may allow a treatment in the context of the Calculus of Variations by using all the tools and techniques of this discipline. This perspective have already been explored in [2] for one-dimensional situations and first order problems and, for instance, in [12] for vector, higher dimensional problems.

The interpretation of our problem as looking for the optimal shape of a bending beam has also received some attention recently. We believe that our approach is however more transparent and direct, and some delicate technical issues can be resolved in an elementary way (see [6] and references therein). We will also see 
how our results can be adapted to treat a somewhat more general situation where we replace the third power occuring in (1.1) by a non-vanishing real power $s$.

The main contribution here is the somewhat surprising result, contrary to the higher dimensional situation ([3], [11]), that optimal designs exist. It is however true that existence of optimal solutions in simplified one-dimensional situations occurs in a variety of situations (see for instance [1], [7], [10]). This existence (Section 3) is achieved by a suitable reformulation as a variational problem (Section 2) to which classical techniques can be applied. In the final sections, optimality conditions are also explored and numerical implementations based on optimality are shown.

\section{A variational reformulation}

Let $f$ be a primitive of a primitive of $F$, i.e.

$$
f(x)=\int_{0}^{x} \int_{0}^{t} F(s) d s d t
$$

then the differential law can be reinterpreted by writing

$$
h(x)^{s} y^{\prime \prime}(x)=f(x)+l(x)
$$

where $l(x)$ is a linear function in $(0,1)$. The underlying idea for our reformulation is to consider (2.1) as a substitute of the differential equation and as a way to relate the pairs $(y, l)$ with the design $h$. In addition, by performing an integration by parts twice on the cost functional and bearing in mind the boundary conditions on $y$, it is straightforward to realize

$$
J(h)=\int_{0}^{1} f(x) y^{\prime \prime}(x) d x
$$

or even further

$$
J(h)=\int_{0}^{1}(f(x)+l(x)) y^{\prime \prime}(x) d x
$$

for any linear function $l(x)$. More precisely, consider the set

$$
\Delta=\left\{(x, \lambda, \xi) \in \mathbf{R}^{3}: h^{s} \xi=f(x)+\lambda \text { for some } h \in\left[h_{-}, h_{+}\right]\right\},
$$


and define the density

$$
\varphi: \Delta \rightarrow \mathbf{R}^{*}=\mathbf{R} \cup\{+\infty\}
$$

by setting

$$
\varphi(x, \lambda, \xi)= \begin{cases}(f(x)+\lambda) \xi, & (x, \lambda, \xi) \in \Delta, \\ +\infty, & \text { else. }\end{cases}
$$

The cost functional for the equivalent variational problem will be

$$
I(l, y)=\int_{0}^{1} \varphi\left(x, l(x), y^{\prime \prime}(x)\right) d x
$$

where $y \in H^{2}(0,1)$, it complies with all the boundary conditions and $l$ is linear. The volume restriction, however, poses some difficulties. Indeed, solving for $h$ in (2.1) leads us to impose

$$
\int_{0}^{1}\left(\frac{f(x)+l(x)}{y^{\prime \prime}(x)}\right)^{1 / s} d x \leq V .
$$

For convenience, let us put

$$
\psi(x, \lambda, \xi)= \begin{cases}\left(\frac{f(x)+\lambda}{\xi}\right)^{1 / s}, & (x, \lambda, \xi) \in \Delta \\ +\infty, & \text { else. }\end{cases}
$$

If the set where $f$ equals a linear function is not negligible, the definition of $\Delta$ forces $y^{\prime \prime}$ to vanish in that same set, and then $h$ can be chosen arbitrarily on the admissible interval $\left[h_{-}, h_{+}\right]$. But that set does not contribute to the cost functional $\left(f^{\prime \prime}=0\right)$ so that we would use as little material as we would be allowed, i.e. we would choose $h(x)=h_{-}$in that set. This possibility has to be compared with other choices when looking for optimal profiles.

Theorem 2.1. Our original optimal design problem is equivalent to the variational problem

$$
\text { Minimize } \quad I(l, y)=\int_{0}^{1} \varphi\left(x, l(x), y^{\prime \prime}(x)\right) d x
$$


subject to

$$
\begin{gathered}
l \text {, linear, } y \in H^{2}(0,1), \quad y(0)=y(1)=y^{\prime}(0)=y^{\prime}(1)=0, \\
\int_{0}^{1} \psi\left(x, l(x), y^{\prime \prime}(x)\right) d x \leq V,
\end{gathered}
$$

in the sense that for any given feasible design $h$, there exists a feasible pair $(l, y)$, and for any such given admissible pair $(l, y)$ there is an admissible design $h$, such that $J(h)=I(l, y)$. In particular the passage from optimal solutions of one problem to optimal solutions of the other is given by

$$
h(x)=\left(\frac{f(x)+l(x)}{y^{\prime \prime}(x)}\right)^{1 / s} .
$$

The proof has almost been indicated, and as a matter of fact, the new variational principle has been set up so that this equivalence is guaranteed.

We would like to clarify the existence-nonexistence of optimal solutions through the analysis of this equivalent variational problem by using the classical tool of the direct method.

\section{Existence of optimal solutions}

It is well-known that there are two main ingredients in the direct method of the Calculus of Variations in order to show existence of optimal solutions: weak lower semicontinuity and coercivity ([8]). Moreover weak lower semicontinuity is equivalent to convexity of the functionals involved in those variables where we have weak but not strong convergence ([13]). We treat those two issues succesively.

We first elucidate the weak lower semicontinuity property for our variational problem. This essentially involves the convexity of the functions $\varphi$ and $\psi$ with respect to $\xi$. Notice that the weak convergence

$$
l_{j} \rightarrow l \quad \text { in } L^{2}(0,1)
$$

when all $l_{j}$ 's are linear implies that $l$ itself is linear and the convergence is indeed strong. This is straightforward. Because of this fact, convexity of $\varphi$ and $\psi$ with respect to $\lambda$ is not enforced nor needed. 
Lemma 3.1. The function $\varphi(x, \lambda, \xi)$ is convex with respect to $\xi$ for any value of $s$ while $\psi(x, \lambda, \xi)$ is convex with respect to $\xi$ provided $s \geq-1$.

Proof. The proof consists in the realization that the set where both $\varphi$ and $\psi$ are finite, i.e. the set $\Delta$, is convex in $\xi$ for fixed $(x, \lambda)$. In fact

$$
\Delta(x, \lambda)=\{\xi \in \mathbf{R}:(x, \lambda, \xi) \in \Delta\}
$$

can be given explicitly as the interval with end-points

$$
\frac{f(x)+\lambda}{h_{-}^{s}} \text { and } \frac{f(x)+\lambda}{h_{+}^{s}} .
$$

In any case this is indeed a convex set. In addition, on such interval the functions $\varphi$ and $\psi$ are convex. On the one hand, $\varphi$ is even linear. On the other, $\psi$ is convex if $s \geq-1$ because in $\Delta f(x)+\lambda$ and $\xi$ must have the same sign.

The weak lower semicontinuity is a direct consequence of the convexity shown in the lemma. This is a standard fact.

Proposition 3.2. The functionals with integrands $\varphi$ and $\psi$ are weak lower semicontinuous with respect to weak convergence in $L^{2}(0,1) \times H^{2}(0,1)$. Namely, if

$$
l_{j} \rightarrow l \quad \text { in } L^{2}(0,1), \quad y_{j} \rightarrow y \quad \text { in } H^{2}(0,1),
$$

then

$$
\begin{aligned}
\liminf _{j \rightarrow \infty} \int_{0}^{1} \varphi\left(x, l_{j}(x), y_{j}^{\prime \prime}(x)\right) d x & \geq \int_{0}^{1} \varphi\left(x, l(x), y^{\prime \prime}(x)\right) d x, \\
\liminf _{j \rightarrow \infty} \int_{0}^{1} \psi\left(x, l_{j}(x), y_{j}^{\prime \prime}(x)\right) d x & \geq \int_{0}^{1} \psi\left(x, l(x), y^{\prime \prime}(x)\right) d x .
\end{aligned}
$$

Coercivity is another main ingredient for the success of the direct method in providing optimal solutions. In our situation, this is immediate because

$$
h_{-}^{s} \xi^{2} \leq(f(x)+\lambda) \xi
$$

if $(x, \lambda, \xi) \in \Delta$. Concerning coercivity for $\lambda$ it is also elementary to obtain

$$
h_{-}^{2 s} \xi^{2} \leq|f(x)+\lambda|^{2}
$$

if $(x, \lambda, \xi) \in \Delta$. The following theorem is a rather routine application of the direct method. 
Theorem 3.3. Our variational problem admits optimal solutions if $s \geq-1$, and consequently, so does our original optimal design problem.

\section{Optimality conditions}

Once the existence of optimal solutions has been rigorously established, we are entitled to pursue the analysis of optimality conditions in order to better understand the features of optimal solutions. Since the analysis that follows does not depend on the particular value of $s$ as long as $s>0$ and the value $s=3$ is particularly important, we have restrict our computations in this section to this value $s=3$, though the same calculations are valid for any such value of $s$.

It turns out that the best way we have found to write optimality conditions down is to go back to the genuine design variable $h$ but keeping the variational form of the problem. In particular we still use (2.1) to write

$$
y^{\prime \prime}(x)=\frac{f(x)+l(x)}{h^{3}(x)},
$$

and thus eliminate the variable $y^{\prime \prime}$ from the variational form of the problem. It is elementary to check that in this way we can write the problem in a new equivalent form as

$$
\text { Minimize } \int_{0}^{1} f(x) \frac{f(x)+l(x)}{h^{3}(x)} d x
$$

subject to

$$
\begin{gathered}
\int_{0}^{1} \frac{f(x)+l(x)}{h^{3}(x)} d x=0 \\
\int_{0}^{1} x \frac{f(x)+l(x)}{h^{3}(x)} d x=0 \\
\int_{0}^{1} h(x) d x \leq V \\
h_{-} \leq h(x) \leq h_{+}
\end{gathered}
$$

These two new integral constraints come from the fact that

$$
y^{\prime \prime}(x)=\frac{f(x)+l(x)}{h^{3}(x)}
$$


must comply with the boundary conditions

$$
y(0)=y(1)=y^{\prime}(0)=y^{\prime}(1)=0 .
$$

If we consider the condition $l$ " $=0$ as a "state equation" regarding $l$ as the state and $h$ as the control, it is elementary to apply Pontryaguin's maximum principle to obtain optimality conditions. Indeed, if we let

$$
l_{1}=l, \quad l_{2}=l^{\prime}, \quad l_{1}^{\prime}=l_{2}, \quad l_{2}^{\prime}=0,
$$

the hamiltonian is

$$
H\left(x, l_{1}, l_{2}, p_{1}, p_{2}, h\right)=\frac{\left(f(x)+l_{1}(x)\right)\left(f(x)+\lambda_{1}+\lambda_{2} x\right)}{h^{3}}+\lambda_{3} h+p_{1} l_{2},
$$

where multipliers $\lambda_{i}$ are associated with the three integral constraints and $\lambda_{3} \geq 0$. Indeed $\lambda_{3}>0$ is expected because the optimal $h$ will yield maximum volume $V$. The dynamics for the costates $p_{i}$ are governed by

$$
p_{1}^{\prime}=-\frac{\left(f(x)+\lambda_{1}+\lambda_{2} x\right)}{h^{3}}, \quad p_{2}^{\prime}=-p_{1},
$$

together with the transversality conditions

$$
p_{1}(0)=p_{1}(1)=p_{2}(0)=p_{2}(1)=0 \text {. }
$$

Therefore we can write

$$
p_{1}(x)=-\int_{0}^{x} \frac{\left(f(t)+\lambda_{1}+\lambda_{2} t\right)}{h(t)^{3}} d t
$$

and demand

$$
\int_{0}^{1} \frac{\left(f(t)+\lambda_{1}+\lambda_{2} t\right)}{h(t)^{3}} d t=0 .
$$

Likewise

$$
\begin{gathered}
p_{2}(x)=\int_{0}^{x} \int_{0}^{s} \frac{\left(f(t)+\lambda_{1}+\lambda_{2} t\right)}{h(t)^{3}} d t d s, \\
\int_{0}^{1} \int_{0}^{s} \frac{\left(f(t)+\lambda_{1}+\lambda_{2} t\right)}{h(t)^{3}} d t d s=0 .
\end{gathered}
$$


Integrating by parts in this last integral and bearing in mind (4.1), we conclude

$$
\int_{0}^{1} t \frac{\left(f(t)+\lambda_{1}+\lambda_{2} t\right)}{h(t)^{3}} d t=0 .
$$

(4.1) and (4.2) imply that $l_{1}(x)=\lambda_{1}+\lambda_{2} x$. Therefore the remaining optimality condition enforces $h(x)$ to be the point of attainment of the minimum

$$
\min _{h_{-} \leq h \leq h_{+}}\left(\frac{\left(f(x)+l_{1}(x)\right)^{2}}{h^{3}}+\lambda_{3} h\right) .
$$

This together with the three integral constraints

$$
\begin{aligned}
& \int_{0}^{1} \frac{f(x)+l_{1}(x)}{h^{3}(x)} d x=0 \\
& \int_{0}^{1} x \frac{f(x)+l_{1}(x)}{h^{3}(x)} d x=0 \\
& \int_{0}^{1} h(x) d x=V
\end{aligned}
$$

determines uniquely the optimal $h(x)$ and $l_{1}(x)$, and the multiplier $\lambda_{3}$. Even further if we put

$$
\gamma(t)= \begin{cases}t, & h_{-} \leq t \leq h_{+}, \\ h_{-}, & t \leq h_{-}, \\ h_{+}, & t \geq h_{+},\end{cases}
$$

then

$$
h(x)=\gamma\left(\left(3 / \lambda_{3}\right)^{1 / 4} \sqrt{\left|f(x)+l_{1}(x)\right|}\right) .
$$

This information already yields interesting qualitative properties about optimal designs and how they can be found. If we rewrite this last formula in terms of

$$
g(x)=\left(\frac{3}{\lambda_{3}}\right)^{1 / 2}\left(f(x)+l_{1}(x)\right),
$$

we see that

$$
h(x)=\gamma(\sqrt{|g(x)|})
$$


where

$$
\int_{0}^{1} \frac{g(x)}{h(x)^{3}} d x=\int_{0}^{1} x \frac{g(x)}{h(x)^{3}} d x=0, \quad \int_{0}^{1} \gamma(\sqrt{|g(x)|}) d x=V .
$$

Notice that $F=\left(\lambda_{3} / 3\right)^{1 / 2} g^{\prime \prime}(x)$ implies that $g$ is smooth. In particular, we see that in the region $h_{-}<h(x)<h_{+}, h$ must be the square root of $g$. For instance, if $F$ is constant, then transition from $h_{-}$to $h_{+}$must take place by arcs of the square root. We will see this behavior in the numerical computations of the next section. These computations are based on the optimality information in this section.

\section{Numerical computations}

The numerical simulations that follow have been implemented by using in an elementary way the equilibrium conditions (4.3) and (4.4). The algorithm used is an elementary fixed point scheme transforming the multipliers $\lambda_{i}, i=1,2,3$, by utilizing (4.4). We have chosen two typical load regimes: the uniformly distributed constant load and the point Dirac delta load. Several simulations have been conducted in each case

1. for different values of the volume $V$;

2. for different values of $h_{-}$;

3. for different values of the power $s$.

For the uniform distributed constant vertical load $F$, numerical results appear in Figures 1, 2, and 3, for the varying volume, varying $h_{-}$and different powers $s$, respectively.

For the point Dirac delta load situated in the middle point of the beam, numerical optimal profiles, for the same three above situations, appear in Figures 4, 5, and 6.

\section{Acknowledgements}

This work is part of the project BFM2001-0738 (Spain). 


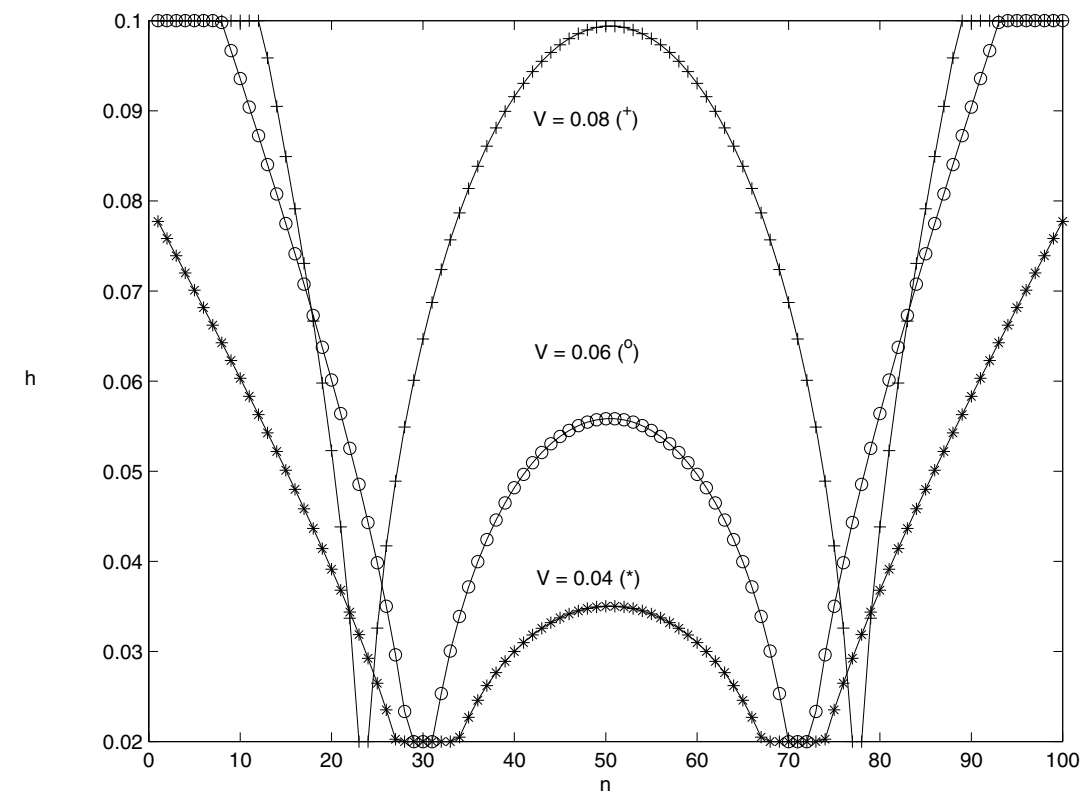

Figure 1 - Optimal profile for different values of $V$.

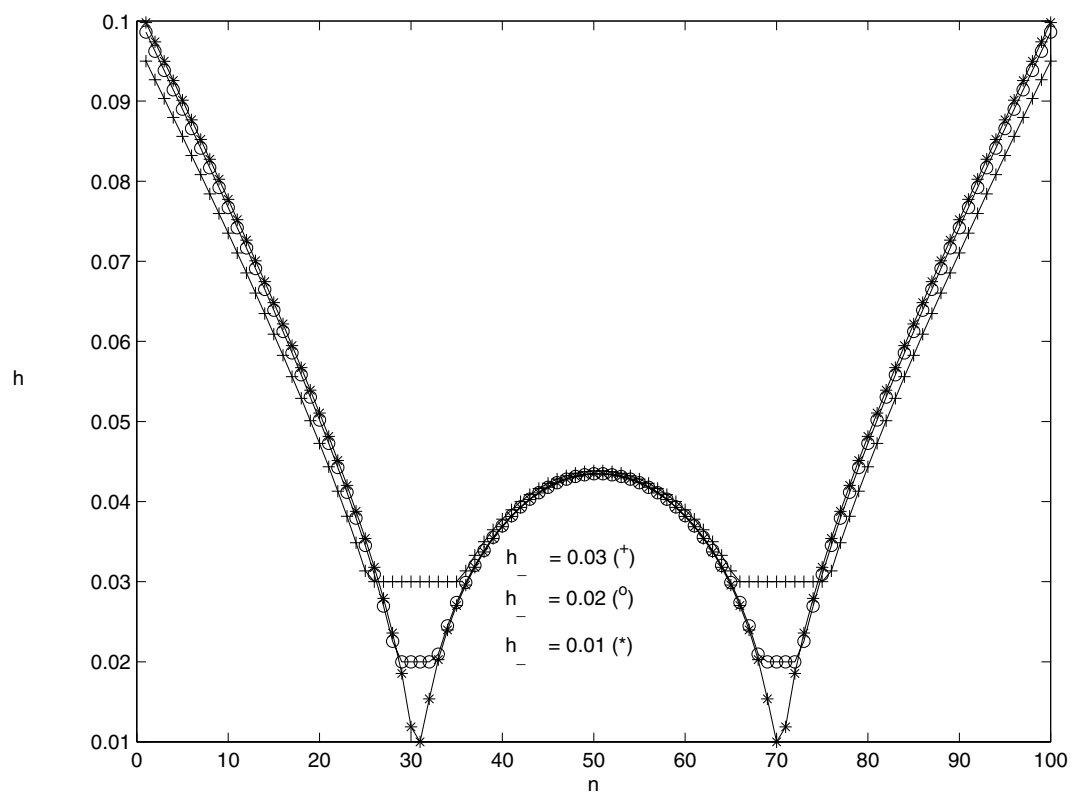

Figure 2 - Optimal profile for different values of $h_{-}$. 


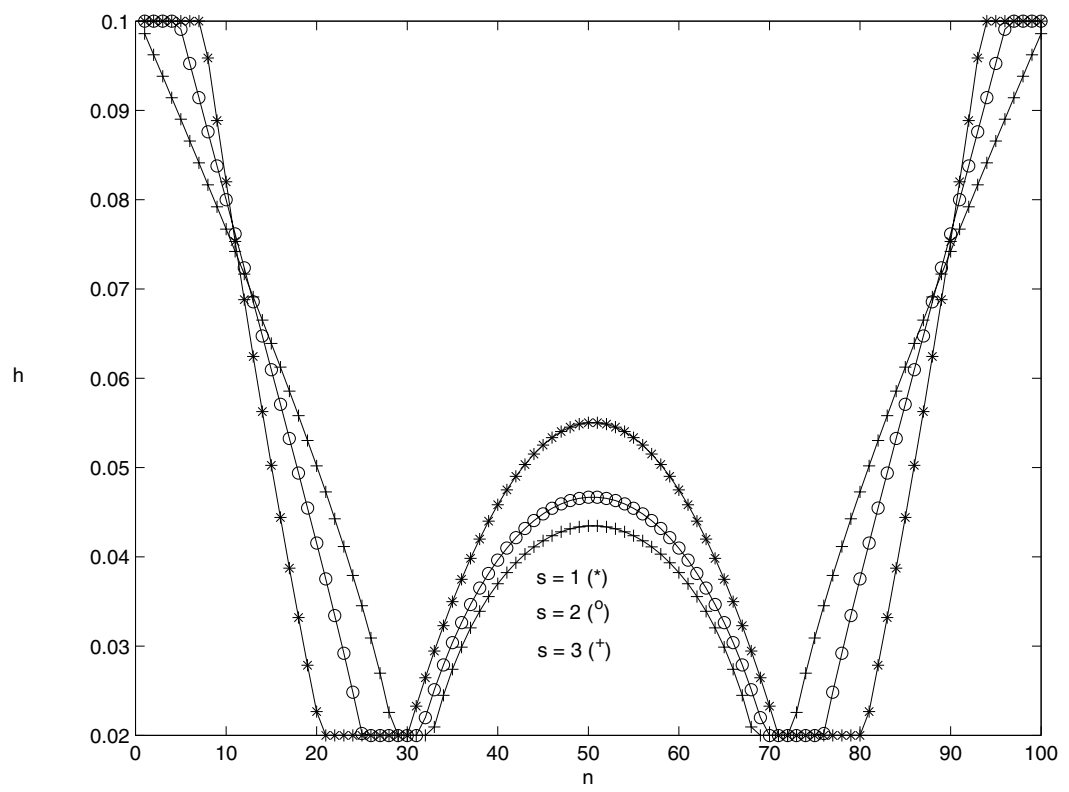

Figure 3 - Optimal profile for different powers $s$.

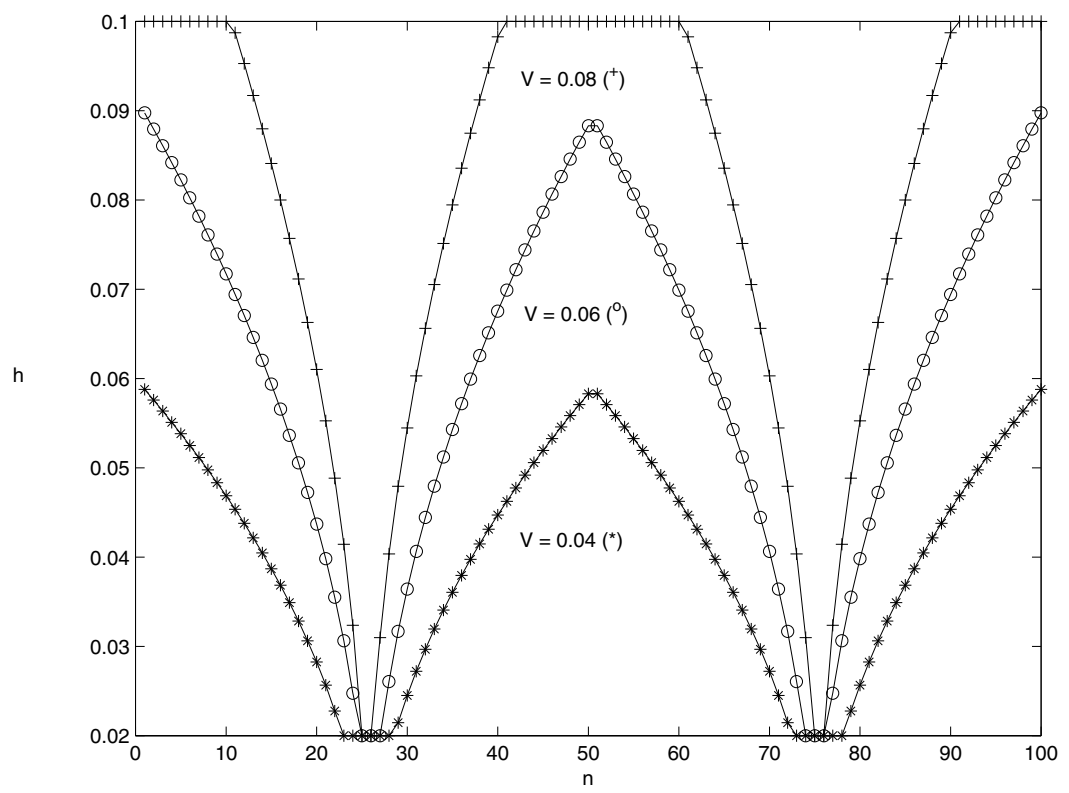

Figure 4 - Optimal profile for different values of $V$. 


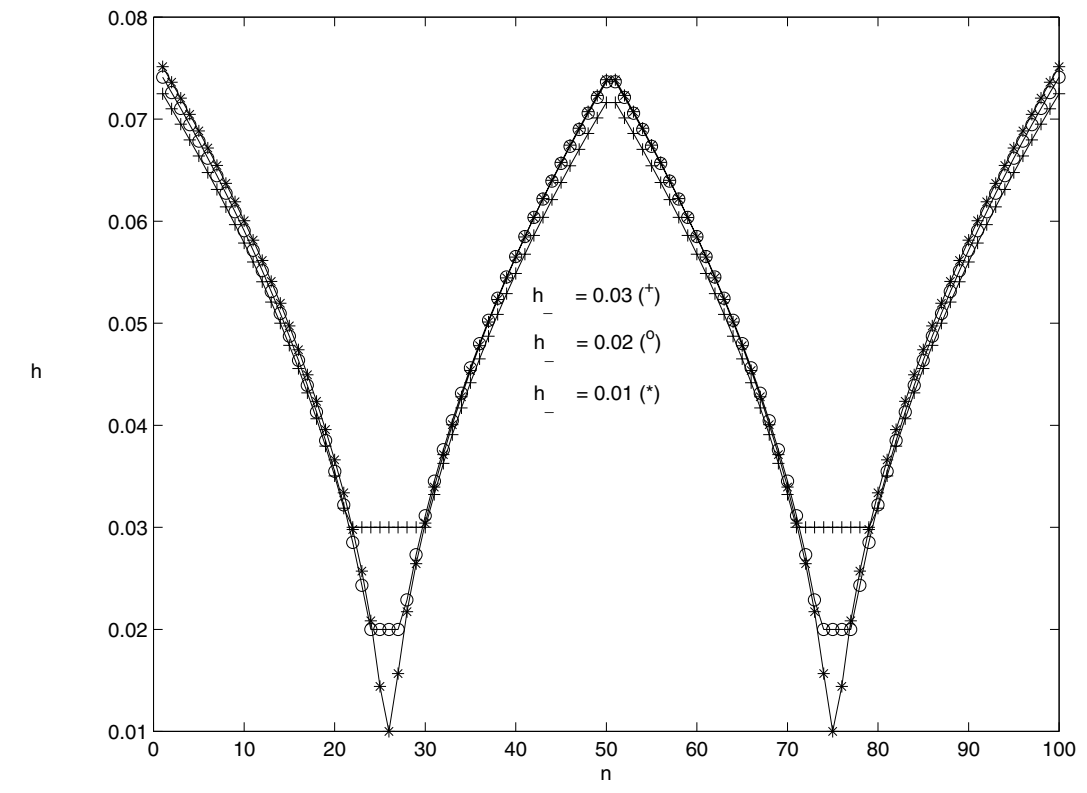

Figure 5 - Optimal profile for different values of $h_{-}$

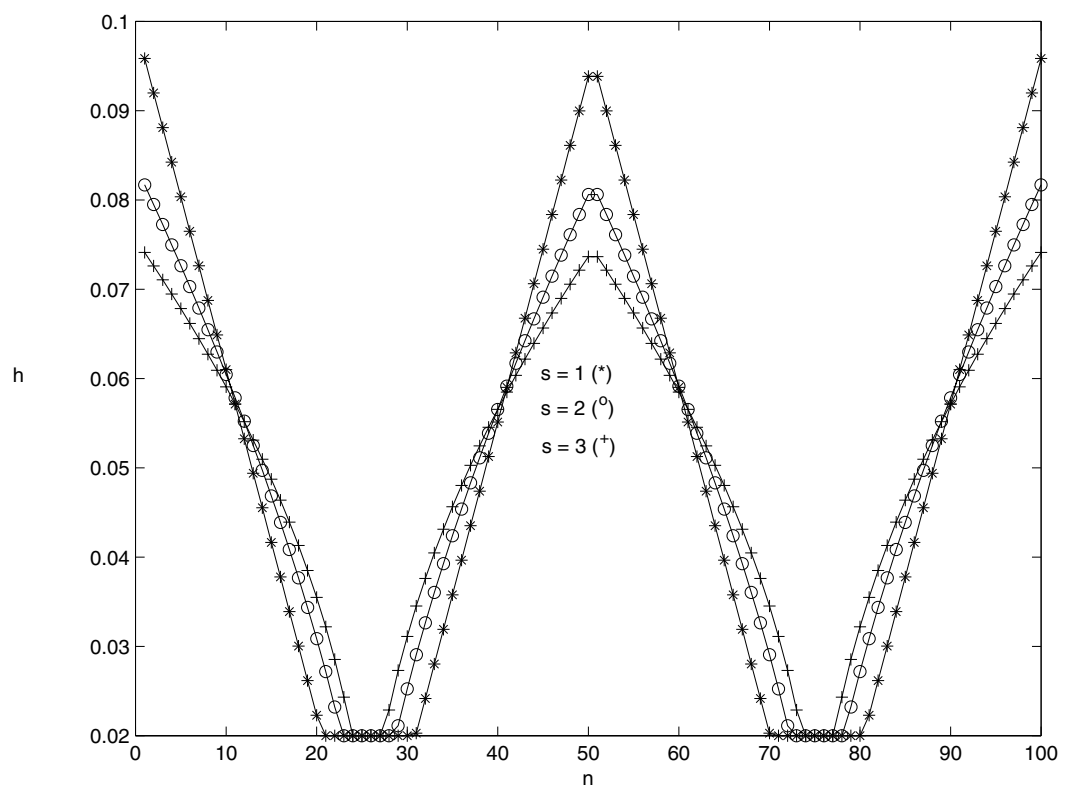

Figure 6-Optimal profile for different powers $s$. 


\section{REFERENCES}

[1] Barnes, D.C., Extremal problems for eigenvalues functionals, SIAM J. Math. Anal., 19(1988), $1151-1161$.

[2] Bellido, J.C. and Pedregal, P., Optimal design via variational principles: the one-dimensional case, J. Math. Pures Appl., 80 (2001), 245-261.

[3] Bonnetier, E. and Conca, C., Relaxation totale d'un problème d'optimisation de plaques, CRAS Paris, 317 (1993), 931-936.

[4] Bonnetier, E. and Conca, C., Approximation of Young measures by functions and application to a problem of optimal design for plates with variable thickness, Proc. Roy. Soc. Edin., A, 124 (1994), 399-422.

[5] Bonnetier, E. and Vogelius, M., Relaxation of a compliance functional for a plate optimization problem, Applications of Multiple Scaling in Mechanics, (P.G. Ciarlet and E. SánchezPalencia, eds.), Masson, 31-53, (1987).

[6] Bratus, A.S. and Posvyanskii, V.P., The optimum shape of a bending beam, J. Appl. Maths. Mechs., 64 (2000), 993-1004.

[7] Cox, S. and Overton, M., On the optimal design of columns against buckling, SIAM J. Math. Anal., 23 (1992), 287-325.

[8] Dacorogna, B., Direct methods in the Calculus of Variations, Springer, (1989).

[9] Kohn, R.V. and Vogelius, M., Thin plates with varying thickness, and their relation to structural optimization, Homogenization and Effective Moduli of Materials and Media, IMA Volumes 1, (Ericksen, J., Kinderlehrer, D., Kohn, R., Lions, J.L., eds), Springer-Verlag, 126-149, (1986).

[10] Muñoz, J., (submitted).

[11] Muñoz, J. and Pedregal, P., On the relaxation of an optimal design problem for plates, Asympt. Anal., 162 (1996), 125-140.

[12] Pedregal, P., Optimal design and constrained quasiconvexity, SIAM J. Math. Anal., 32 (2000), 854-869.

[13] Pedregal, P., Parametrized Measures and Variational Principles, Birkhäuser, Basel, (1997). 